\title{
ON PRIME NONPRIMITIVE VON NEUMANN REGULAR ALGEBRAS
}

\author{
GENE ABRAMS, JASON P. BELL, AND KULUMANI M. RANGASWAMY
}

\begin{abstract}
Let $E$ be any directed graph, and $K$ any field. We classify those graphs $E$ for which the Leavitt path algebra $L_{K}(E)$ is primitive. As a consequence, we obtain classes of examples of von Neumann regular prime rings which are not primitive.
\end{abstract}

\section{INTRODUCTION}

The structure of prime and primitive algebras has long been the focus of much attention. The spark for much of the interest in such structures was a question posed by Kaplansky [16, p. 2]: "Is a regular prime ring necessarily primitive?" This question was, in its time, "... the major question in the theory of von Neumann regular rings" ([26, p. 308]). An example of such a ring (a very clever but somewhat ad hoc construction of a specific group algebra) was first given in 1977 by Domanov (see [11; see also 24] for a more complete account).

The original construction in 2004 of the Leavitt path algebras was spawned by an appropriate melding of the ideas underlying the construction of algebras presented by W. Leavitt in 1962 (the now so-called Leavitt algebras; see [20]) with the ideas underlying the construction of the analytic structures known as graph $\mathrm{C}^{*}$-algebras (see e.g. 25]). The graph $\mathrm{C}^{*}$-algebras have genesis in the so-called Cuntz $\mathrm{C}^{*}$ algebras constructed by J. Cuntz in 1977 (see [10]).

It is fair to say that both Leavitt and Cuntz were motivated to fashion their appropriately-named structures by specific questions in their respective disciplines: Leavitt sought a general algebraic construction which would produce rings affording isomorphisms between free modules of differing finite ranks, while Cuntz sought an explicit example of a separable simple infinite $\mathrm{C}^{*}$-algebra. Subsequently, many examples of graph $\mathrm{C}^{*}$-algebras that have been built were motivated by a specific question in the theory of $\mathrm{C}^{*}$-algebras (see e.g. [25, Proposition 2.12 and Corollary $7.19]$ ); an analogous statement for Leavitt path algebras holds as well (see e.g. [3. Theorem 4.2]).

The main purpose of the current article is to continue this tradition. To wit: we give a systematic answer to the aforementioned question of Kaplansky by building Leavitt path algebras possessing the desired properties. Specifically, we provide necessary and sufficient conditions on an arbitrary directed graph $E$ which ensure

Received by the editors May 16, 2011 and, in revised form, April 4, 2012 and May 15, 2012.

2010 Mathematics Subject Classification. Primary 16G20, 05E10.

Key words and phrases. Leavitt path algebra, prime ring, primitive ring, countable separation property.

The first author was partially supported by the U.S. National Security Agency under grant number H89230-09-1-0066.

The second author was supported by NSERC grant 31-611456. 
that the Leavitt path algebra $L_{K}(E)$ is prime (Theorem 2.4) and which ensure that $L_{K}(E)$ is primitive (Theorem [5.7). (See Section 2 for the precise definition of these structures.) As we shall see, the primeness condition in the general case exactly matches the primeness condition given in [7] for row-finite graphs. On the other hand, the primitivity of $L_{K}(E)$ in the general case requires an additional condition (the "Countable Separation Property") on the graph $E$, a condition which is automatically satisfied for row-finite graphs $E$ having prime $L_{K}(E)$.

If $u$ and $v$ are vertices in the graph $E$, we write $u \geq v$ in the case $u=v$ or there is a directed path in $E$ whose source vertex is $u$ and range vertex is $v$. If $c=e_{1} e_{2} \cdots e_{n}$ is a cycle in $E$, then an exit for $c$ is an edge $f$ which is not equal to any of the $e_{i}$, but whose source vertex is equal to the source vertex of one of the $e_{i}$. Since any Leavitt path algebra is isomorphic to its opposite ring (see Proposition 2.2), left and right primitivity coincide for this class of rings. With this information in hand, we now present our main result (Theorem [5.7), in which we establish an easy-to-verify list of properties of $E$ which taken together are equivalent to the primitivity of $L_{K}(E)$.

Theorem. Let $E$ be an arbitrary graph, and $K$ any field. Then $L_{K}(E)$ is primitive if and only if

(i) for every pair of vertices $u, v$ in $E$ there exists a vertex $w$ in $E$ for which $u \geq w$ and $v \geq w$

(ii) every cycle in $E$ has an exit, and

(iii) there exists a countable set $S$ of vertices in $E$ for which, for each vertex $u$ in $E$, there exists $w \in S$ for which $u \geq w$.

These conditions on $E$ are called, respectively, downward directedness, Condition (L), and the Countable Separation Property.

Conditions on the graph $E$ which imply the von Neumann regularity of $L_{K}(E)$ were established in [2]. With such information in hand, Theorems 2.4 and 5.7 then immediately allow us to produce a bumper crop of examples of the type posed by Kaplansky: prime nonprimitive von Neumann regular algebras. Four such infinite classes of algebras (two of which consist of unital algebras) are presented in Section 6 .

\section{LeAvitt PATH Algebras, AND the PRIMENESS THEOREM}

We begin by giving a condensed reminder of the germane definitions. For a more complete description and discussion, see for example [26] and [1]. A ring $R$ is called prime if the product of any two nonzero two-sided ideals of $R$ is again nonzero, left primitive if $R$ admits a simple faithful left $R$-module, and von Neumann regular (or sometimes more concisely regular) in the case for each $a \in R$ where there exists $x \in R$ for which $a=a x a$. It is easy to show that any primitive ring is prime. Furthermore, in a prime ring, the intersection of any two nonzero two-sided ideals is nonzero, as the intersection contains the product.

A (directed) graph $E=\left(E^{0}, E^{1}, r, s\right)$ consists of two sets $E^{0}, E^{1}$ and functions $r, s: E^{1} \rightarrow E^{0}$. The elements of $E^{0}$ are called vertices and the elements of $E^{1}$ edges. The sets $E^{0}$ and $E^{1}$ are allowed to be of arbitrary cardinality. A path $\mu$ in a graph $E$ is a finite sequence of edges $\mu=e_{1} \ldots e_{n}$ such that $r\left(e_{i}\right)=s\left(e_{i+1}\right)$ for $i=1, \ldots, n-1$. In this case, $s(\mu):=s\left(e_{1}\right)$ is the source of $\mu, r(\mu):=r\left(e_{n}\right)$ is the range of $\mu$, and $n:=\ell(\mu)$ is the length of $\mu$. We view the elements of $E^{0}$ as paths of 
length 0 . If $\mu=e_{1} \ldots e_{n}$ is a path in $E$, and if $v=s(\mu)=r(\mu)$ and $s\left(e_{i}\right) \neq s\left(e_{j}\right)$ for every $i \neq j$, then $\mu$ is called a cycle based at $v$. A graph which contains no cycles is called acyclic. An edge $e$ is an exit for a path $\mu=e_{1} \ldots e_{n}$ if there exists $i$ such that $s(e)=s\left(e_{i}\right)$ and $e \neq e_{i}$. If $s^{-1}(v)$ is a finite set for every $v \in E^{0}$, then the graph $E$ is called row-finite.

Definition 2.1. Let $E$ be any directed graph, and $K$ any field. The Leavitt path $K$-algebra $L_{K}(E)$ of $E$ with coefficients in $K$ is the $K$-algebra generated by a set $\left\{v \mid v \in E^{0}\right\}$, together with a set of variables $\left\{e, e^{*} \mid e \in E^{1}\right\}$, which satisfy the following relations:

(V) $v w=\delta_{v, w} v$ for all $v, w \in E^{0} \quad$ (i.e., $\left\{v \mid v \in E^{0}\right\}$ is a set of orthogonal idempotents).

(E1) $s(e) e=e r(e)=e$ for all $e \in E^{1}$.

(E2) $r(e) e^{*}=e^{*} s(e)=e^{*}$ for all $e \in E^{1}$.

(CK1) $e^{*} e^{\prime}=\delta_{e, e^{\prime}} r(e)$ for all $e, e^{\prime} \in E^{1}$.

(CK2) $v=\sum_{\left\{e \in E^{1} \mid s(e)=v\right\}} e e^{*}$ for every vertex $v \in E^{0}$ having $1 \leq\left|s^{-1}(v)\right|<\infty$.

We let $r\left(e^{*}\right)$ denote $s(e)$, and we let $s\left(e^{*}\right)$ denote $r(e)$. If $\mu=e_{1} \ldots e_{n}$ is a path, then we denote by $\mu^{*}$ the element $e_{n}^{*} \ldots e_{1}^{*}$ of $L_{K}(E)$.

Many well-known algebras arise as the Leavitt path algebra of a graph. For example, the classical Leavitt $K$-algebra $L_{K}(1, n)$ for $n \geq 2$; the full $n \times n$ matrix algebra $\mathrm{M}_{n}(K)$ over $K$; and the Laurent polynomial algebra $K\left[x, x^{-1}\right]$ arise, respectively, as the Leavitt path algebras of the "rose with $n$ petals" graph $R_{n}(n \geq 2)$; the oriented line graph $A_{n}$ having $n$ vertices; and the "one vertex, one loop" graph $R_{1}$ pictured here:

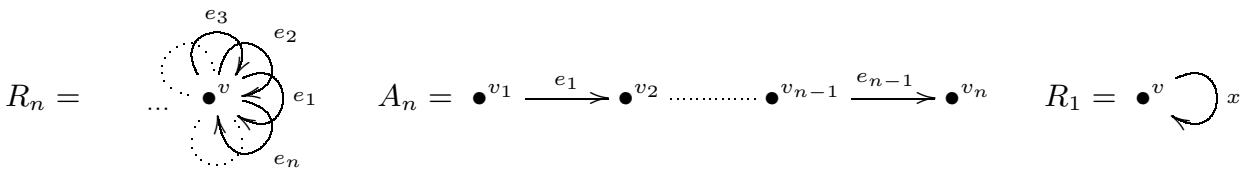

A (possibly nonunital) ring $R$ is called a ring with local units in the case where for each finite subset $S \subseteq R$ there is an idempotent $e \in R$ with $S \subseteq e R e$. If $E$ is a graph for which $E^{0}$ is finite, then we have that $\sum_{v \in E^{0}} v$ is the multiplicative identity in $L_{K}(E)$; otherwise, $L_{K}(E)$ is a ring with a set of local units consisting of sums of distinct vertices. Conversely, if $L_{K}(E)$ is unital, then $E^{0}$ is finite.

The Leavitt path algebra $L_{K}(E)$ is a $\mathbb{Z}$-graded $K$-algebra, spanned as a $K$-vector space by $\left\{p q^{*} \mid p, q\right.$ are paths in $\left.E\right\}$. (Recall that the elements of $E^{0}$ are viewed as paths of length 0 , so that this set includes elements of the form $v$ with $v \in E^{0}$.) That is, each element of $L_{K}(E)$ may be expressed (not necessarily uniquely) in the form

$$
\sum_{i=1}^{n} k_{i} \alpha_{i} \beta_{i}^{*},
$$

where $k_{i} \in K$, and $\alpha_{i}, \beta_{i}$ are paths in $E$ for which $r\left(\alpha_{i}\right)=r\left(\beta_{i}\right)$. We say that an element of $L_{K}(E)$ expressed in this way is in standard form. In particular, for each $n \in \mathbb{Z}$, the degree $n$ component $L_{K}(E)_{n}$ is spanned by the set $\left\{p q^{*}\right.$ | $p$ and $q$ are paths in $E$, with length $(p)-\operatorname{length}(q)=n\}$.

Throughout, the word "graph" will mean "directed graph", and the word "ideal" will mean "two-sided ideal" (unless otherwise indicated). If $u, v$ are vertices in the graph $E$, we write $u \geq v$ in the case where there is a path $p$ in $E$ for which 
$s(p)=u, r(p)=v$. If $p$ and $q$ are paths in $E$, we say $p$ is an initial subpath of $q$ in the case where there is a path $p^{\prime}$ in $E$ for which $q=p p^{\prime}$. If $I$ is an ideal of $L_{K}(E)$, and $u, v \in E^{0}$ for which $u \in I$ and $u \geq v$, then $v=p^{*} p=p^{*} u p \in I$.

We note a basic result about Leavitt path algebras, thereby reiterating an observation which was made in [8, Introduction].

Proposition 2.2. Let $E$ be an arbitrary graph, and $K$ any field. Then there is a $K$-algebra isomorphism $L_{K}(E)^{o p} \cong L_{K}(E)$.

Proof. The map $\varphi: L_{K}(E) \rightarrow L_{K}(E)$ defined on the standard form of elements by setting

$$
\varphi: \sum_{i=1}^{n} k_{i} \alpha_{i} \beta_{i}^{*} \mapsto \sum_{i=1}^{n} k_{i} \beta_{i} \alpha_{i}^{*}
$$

is a $K$-linear involution on $L_{K}(E)$ (see e.g. [27, Remark 3.4]). The result then follows immediately from general ring-theoretic properties (see e.g. [12, p. 47]).

As a consequence of this result, throughout the sequel we drop the left/right designation, and simply refer to the primitivity of $L_{K}(E)$. Two key properties of a graph $E$ will play a significant role here.

Definition 2.3. Let $E$ be any graph.

(1) $E$ is downward directed in the case where for every pair of vertices $v, w$ in $E$, there is a vertex $u$ in $E$ such that $v \geq u$ and $w \geq u$.

(2) E satisfies Condition ( $L)$ in the case where every cycle in $E$ has an exit.

We note that the downward directed property is also called Condition (MT3) throughout the literature. In 6, Proposition 5.6], necessary and sufficient conditions on the row-finite graph $E$ are presented which ensure that the Leavitt path algebra $L_{K}(E)$ is prime. (As is the case with many results in this area, the structure of the field $K$ plays no role in these conditions.) We extend this result to arbitrary graphs $E$, while simultaneously giving a somewhat more streamlined argument.

Theorem 2.4. Let $E$ be an arbitrary graph, and $K$ any field. Then $L_{K}(E)$ is a prime ring if and only if $E$ is downward directed.

Proof. Suppose $R=L_{K}(E)$ is a prime ring. Let $v, w \in E^{0}$. Since the ideals $R v R$ and $R w R$ are nonzero, $R v R w R$, and hence $v R w$, are nonzero. This means $v \alpha \beta^{*} w \neq 0$ for some paths $\alpha, \beta$ in $E$, which in particular gives $s(\alpha)=v$ and $s(\beta)=w$. Then $u=r(\alpha)=r(\beta)$ is a vertex which yields the downward directedness property of $E$.

Now suppose that $E$ is downward directed. Since $L_{K}(E)$ is graded by the ordered group $\mathbb{Z}$, by [23, Proposition II.1.4] we see that, to establish the primeness of $L_{K}(E)$, we need only show that $I J \neq\{0\}$ for any pair $I, J$ of nonzero graded ideals of $L_{K}(E)$. By [15, Theorem 3.2], each nonzero graded ideal of $L_{K}(E)$ contains a vertex. Let $v \in I \cap E^{0}$ and $w \in J \cap E^{0}$. By downward directedness there is a vertex $u$ such that $v \geq u$ and $w \geq u$. But then $u \in I$ and $u \in J$, so that $0 \neq u=u^{2} \in I J$, as desired.

We note that M. Siles Molina has also independently achieved the result of Theorem 2.4 


\section{Primitive Leavitt path algebras: The row-Finite case}

In this section we present necessary and sufficient conditions on a row-finite graph $E$ which ensure that the Leavitt path algebra $L_{K}(E)$ is primitive.

We begin by stating four very useful results. The first of these allows us to easily pass primitivity from unital to nonunital rings.

Lemma 3.1 ([19, Lemmas 1 and 2]). Let $K$ be any field, and let $R$ be a prime $K$-algebra. Then there exists a prime unital $K$-algebra $R_{1}$ into which $R$ embeds as an ideal. Furthermore, $R_{1}$ is right (resp., left) primitive if and only if $R$ is right (resp., left) primitive.

Proposition 3.2. Let $E$ be an arbitrary graph which satisfies Condition (L), and let $I$ be any nonzero two-sided ideal of $L_{K}(E)$. Then there exists a vertex $w$ for which $w \in I$.

Proof. This result appears as Corollary 3.3 in [4. Although the standing hypotheses made throughout [4] is that all graphs are row-finite, the proof of [4, Proposition 3.1] and its consequent Corollary 3.3 are easily seen to hold verbatim for arbitrary graphs.

Proposition 3.3 (14, Theorem 1] or [18, Lemma 11.28]). A unital ring $R$ is left primitive if and only if there is a left ideal $M \neq R$ of $R$ such that for every nonzero two-sided ideal $I$ of $R, M+I=R$.

The fourth and final preliminary result is a slight modification of a powerful theorem established by Fisher and Snider in [13. The proof presented here invokes some of the important ideas utilized in the proof of [13, Theorem 1.1].

Proposition 3.4. Let $R$ be a unital ring. Suppose $R$ contains a countable set of nonzero idempotents $\left\{e_{i} \mid i \in \mathbb{N}\right\}$ for which $e_{i} e_{j}=e_{j}$ (resp., $e_{j} e_{i}=e_{j}$ ) for all $i \leq j$, and for which each nonzero two-sided ideal of $R$ contains some $e_{i}$. Then $R$ is left (resp., right) primitive.

Proof. We prove the left primitivity statement (the proof of the right primitivity statement being completely analogous). The given condition easily yields that $\left(1-e_{i}\right)\left(1-e_{j}\right)=1-e_{i}$ for all $i \leq j$, so that $R\left(1-e_{i}\right) \subseteq R\left(1-e_{j}\right)$ for $i \leq j$; thus $M=\bigcup_{i=1}^{\infty} R\left(1-e_{i}\right)$ is a left ideal of $R$. Just suppose $1 \in M$; then there exist $n \in \mathbb{N}$ and $r \in R$ for which $1=r\left(1-e_{n}\right)$. But then multiplying this equation on the right by $e_{n}$ gives $e_{n}=0$, a contradiction. Thus $1 \notin M$, so $M \neq R$. Now suppose $I$ is a nonzero two-sided ideal of $R$. Then by hypothesis $I$ contains some $e_{i}$, so that $M+I$ contains $\left(1-e_{i}\right)+e_{i}=1$, so that $M+I=R$. Now invoking Proposition 3.3 completes the proof.

Using these four results, we are already in a position to establish the row-finite case. As it turns out, we will also be able to establish the row-finite result as a specific case of Theorem 5.7. However, the argument in the row-finite case provides clarification and insight to the general case, so we present it separately.

Theorem 3.5. Let $E$ be a row-finite graph, and $K$ any field. Then $R=L_{K}(E)$ is primitive if and only if

(i) $E$ is downward directed, and

(ii) E satisfies Condition (L). 
Proof. First, suppose $E$ satisfies the two conditions. By Theorem 2.4, downward directedness yields that $L_{K}(E)$ is prime. Using Lemma 3.1, we embed $L_{K}(E)$ as a two-sided ideal in a prime $K$-algebra $L_{K}(E)_{1}$, and establish the primitivity of $L_{K}(E)$ by establishing the primitivity of $L_{K}(E)_{1}$. Let $v$ be any vertex in $E$, and let $T(v)=\left\{u \in E^{0} \mid v \geq u\right\}$. Since $E$ is row-finite, the set $T(v)$ is at most countable. So we may label the elements of $T(v)$ as $\left\{v_{1}, v_{2}, \ldots\right\}$. We inductively define a sequence $\lambda_{1}, \lambda_{2}, \ldots$ of paths in $E$ for which, for each $i \in \mathbb{N}$,

(1) $\lambda_{i}$ is an initial subpath of $\lambda_{j}$ whenever $i \leq j$, and

(2) $v_{i} \geq r\left(\lambda_{i}\right)$

To do so, define $\lambda_{1}=v_{1}$. Now suppose $\lambda_{1}, \ldots, \lambda_{n}$ have been defined with the indicated properties for some $n \in \mathbb{N}$. By downward directedness, there is a vertex $u_{n+1}$ in $E$ for which $r\left(\lambda_{n}\right) \geq u_{n+1}$ and $v_{n+1} \geq u_{n+1}$. Let $p_{n+1}$ be a path from $r\left(\lambda_{n}\right)$ to $u_{n+1}$, and define $\lambda_{n+1}=\lambda_{n} p_{n+1}$. Then $\lambda_{n+1}$ is clearly seen to have the desired properties.

Since $\lambda_{i}$ is a subpath of $\lambda_{t}$ for all $i \leq t$, we get that

$$
\lambda_{i} \lambda_{i}^{*} \lambda_{t} \lambda_{t}^{*}=\lambda_{t} \lambda_{t}^{*} \text { for each pair of positive integers } i \leq t .
$$

Furthermore, each $\lambda_{i} \lambda_{i}^{*}$ is nonzero.

We claim that every nonzero two-sided ideal $I$ of $L_{K}(E)_{1}$ contains some $\lambda_{i} \lambda_{i}^{*}$. Since $L_{K}(E)_{1}$ is prime and $L_{K}(E)$ embeds in $L_{K}(E)_{1}$ as a two-sided ideal, we have that $I \cap L_{K}(E)$ is a nonzero two-sided ideal of $L_{K}(E)$. So Condition $(\mathrm{L})$ on $E$, together with Proposition [3.2, imply that $I$ contains some vertex, call it $w$. By downward directedness there exists $u \in E^{0}$ for which $v \geq u$ and $w \geq u$. But $v \geq u$ gives by definition that $u=v_{n}$ for some $n \in \mathbb{N}$, so that $w \geq v_{n}$. By the construction of the indicated sequence of paths $v_{n} \geq r\left(\lambda_{n}\right)$, so that there is a path $q$ in $E$ for which $s(q)=w$ and $r(q)=r\left(\lambda_{n}\right)$. Since $w \in I$ this gives $r\left(\lambda_{n}\right) \in I$, so that $\lambda_{n} \lambda_{n}^{*}=\lambda_{n} r\left(\lambda_{n}\right) \lambda_{n}^{*} \in I$, as desired.

Thus $M$ satisfies the conditions of Proposition 3.4, which yields the left primitivity of $L_{K}(E)_{1}$, and with it, by Lemma 3.1, the primitivity of $L_{K}(E)$.

Conversely, suppose $R=L_{K}(E)$ is primitive. Since $R$ is then in particular a prime ring, $E$ is downward directed by Theorem 2.4 We argue by contradiction that $E$ has Condition (L) as well, for, if not, there is a cycle $c$ based at a vertex $v$ in $E$ having no exits. But then by [5, Lemma 1.5], the nonzero corner ring $v R v \cong K\left[x, x^{-1}\right]$ is not primitive (as a commutative primitive ring must be a field). (We note that the standing hypotheses throughout [5] require that the graph $E=\left(E^{0}, E^{1}\right)$ satisfies that both sets $E^{0}$ and $E^{1}$ are countable, but the proof of [5. Lemma 1.5] is easily seen to be valid verbatim for graphs of arbitrary cardinality.) Since a nonzero corner of a primitive ring must again be primitive (for if $M$ is a faithful simple left $R$-module and $0 \neq v=v^{2} \in R$, then $v M$ is a faithful simple left $v R v$-module), we reach the desired contradiction, and the result follows.

Remark 3.6. The result appearing directly above as Theorem 3.5 in fact appears as Theorem 4.6 of [7]. However, the proof of [7, Theorem 4.6] relies on [7, Propositions 4.4 and 4.5], and these latter two results are in fact incorrect. Briefly paraphrased, the statement of [7. Proposition 4.4(i)] asserts that if $u \geq v$ are vertices, then the sets $S_{u}$ and $S_{v}$ of isomorphism classes of simple factors of $u L_{K}(E)$ and $v L_{K}(E)$ (respectively) are equal, and, moreover, [7. Proposition 4.4(ii)] claims that the simple factor $u L_{K}(E) / J$ is isomorphic to $v L_{K}(E) / \alpha^{*} J$ (where $\alpha$ is a path from $u$ to $v$ ). Furthermore, [7. Proposition 4.5] proposes that if $u$ is a vertex which emits 
at least two edges, and $M$ is a simple factor of the right ideal $u L_{K}(E)$, then for some edge $e$ emitted by $u$ there is an isomorphism $M \cong r(e) L_{K}(E) / e^{*} L_{K}(E)$. But consider the graph

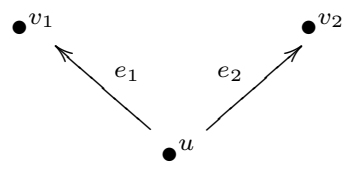

For each $i=1,2$ it is not hard to show that $v_{i}=r\left(e_{i}\right)$ has the property that $v_{i} L_{K}(E)$ is a simple right $L_{K}(E)$-module, and, furthermore, that $e_{i}^{*} L_{K}(E)=v_{i} L_{K}(E)$. Thus the quotients $r\left(e_{i}\right) L_{K}(E) / e_{i}^{*} L_{K}(E)$ are each zero. This clearly violates both of the statements of [7, Proposition 4.4(ii) and 4.5]. Furthermore, in this case we have $\left|S_{u}\right|=2$, while $\left|S_{v_{i}}\right|=1$ for $i=1,2$, thus violating [7, Proposition 4.4(i)].

\section{Primitive Leavitt Path algebras for arbitrary graphs: SUFFICIENT CONDITIONS}

In this section we present three conditions on an arbitrary graph $E$ which, taken together, imply the primitivity of the Leavitt path algebra $L_{K}(E)$ for any field $K$. We start by defining a property of graphs which will play a key role in determining the primitivity of Leavitt path algebras of not necessarily row-finite graphs. As motivation for this property, we observe that the proof of the sufficiency portion of the row-finite case (Theorem 3.5) reveals that, for any vertex $v$ in a downward directed row-finite graph $E$, the set $S=T(v)$ is an at most countable subset of $E^{0}$ such that, for every $w \in E^{0}$, there is some $v_{i} \in S$ for which $w \geq v_{i}$.

Definition 4.1. Let $E$ be a graph and let $S \subseteq E^{0}$. We say that $S$ has the Countable Separation Property (more concisely, CSP) if there is an at most countable set of vertices $C(S)=\left\{v_{1}, v_{2}, \ldots\right\} \subseteq E^{0}$ such that, for every $w \in S$, there is some $v_{i} \in C(S)$ for which $w \geq v_{i}$.

Remark 4.2. Let $E$ be a graph for which $E^{0}$ is at most countable. Then every subset $S$ of $E^{0}$ has CSP (simply use $C(S)=E^{0}$ ).

We now present two examples of classes of graphs which are germane in this context.

Definition 4.3. Let $X$ be any nonempty set, and let $\mathcal{F}(X)$ denote the collection of nonempty finite subsets of $X$. We define the graph $E_{\mathcal{F}(X)}$ as follows:

$$
E_{\mathcal{F}(X)}^{0}=\mathcal{F}(X), \quad E_{\mathcal{F}(X)}^{1}=\left\{e_{A, A^{\prime}} \mid A, A^{\prime} \in \mathcal{F}(X), \text { and } A \varsubsetneqq A^{\prime}\right\},
$$

$s\left(e_{A, A^{\prime}}\right)=A$, and $r\left(e_{A, A^{\prime}}\right)=A^{\prime}$ for each $e_{A, A^{\prime}} \in E_{\mathcal{F}(X)}^{1}$.

Using Remark 4.2 along with standard cardinal arithmetic, we immediately get

Proposition 4.4. Let $X$ be any set, and let $E_{\mathcal{F}(X)}$ be the graph described in Definition 4.3. Then $E_{\mathcal{F}(X)}^{0}$ has CSP if and only if $X$ is at most countable.

Definition 4.5. Let $\kappa>0$ be any ordinal. We define the graph $E_{\kappa}$ as follows:

$$
E_{\kappa}^{0}=\{\alpha \mid \alpha<\kappa\}, \quad E_{\kappa}^{1}=\left\{e_{\alpha, \beta} \mid \alpha, \beta<\kappa, \text { and }\{\alpha<\beta\},\right.
$$

$s\left(e_{\alpha, \beta}\right)=\alpha$, and $r\left(e_{\alpha, \beta}\right)=\beta$ for each $e_{\alpha, \beta} \in E_{\kappa}^{1}$. 
Recall that an ordinal $\kappa$ is said to have countable cofinality in the case where $\kappa$ is the limit of a countable sequence of ordinals strictly less than $\kappa$. For example, any countable ordinal has countable cofinality. The ordinal $\omega_{1}$ does not have countable cofinality, while the ordinal $\omega_{\omega}$ does have this property. With this definition in mind, the following result is clear.

Proposition 4.6. Let $\kappa$ be any ordinal, and let $E_{\kappa}$ be the graph described in Definition 4.5. Then $E_{\kappa}^{0}$ has CSP if and only if $\kappa$ has countable cofinality.

The connection between row-finite graphs and the Countable Separation Property is made evident in the following result.

Lemma 4.7. Let $E$ be a row-finite downward directed graph. Then $E^{0}$ has CSP.

Proof. For any $v \in E^{0}$, consider the set $T(v)=\left\{u \in E^{0} \mid v \geq u\right\}$. As $E$ is row-finite, $T(v)$ is at most countable. But then downward directedness yields that $C(T(v))=E^{0}$, so that $T(v)$ has the desired property.

A reexamination of the proof of the sufficiency direction of Theorem 3.5 reveals that the only properties of the set $T(v)$ utilized therein are that $T(v)$ is countable, and that subsequently $E^{0}$ has the Countable Separation Property with respect to $T(v)$. Indeed, replacing the set $T(v)$ by any set $S$ with respect to which $E^{0}$ has CSP, the proof of Theorem 3.5 immediately yields the sufficiency direction of our main result.

Proposition 4.8. Let $E$ be an arbitrary graph, and $K$ any field. Suppose:

(i) $E$ is downward directed,

(ii) E satisfies Condition (L), and

(iii) $E^{0}$ has the Countable Separation Property.

Then $L_{K}(E)$ is primitive.

In an analogous manner, using Remark 4.2, the proof of Theorem 3.5 also yields the following.

Corollary 4.9. Let $E$ be a graph for which $E^{0}$ is at most countable, and let $K$ be any field. Then $R=L_{K}(E)$ is primitive if and only if

(i) $E$ is downward directed, and

(ii) E satisfies Condition ( $L)$.

5. Primitive Leavitt path algebras for arbitrary graphs: The necessity of the Countable Separation Property of $E^{0}$

We now establish the converse of Proposition 4.8. The heavy lifting needed to achieve this goal will come in showing that if $E$ does not have CSP, then $L_{K}(E)$ is not primitive.

Remark 5.1. Along the way we shall use (most often without explicit mention) various characteristics of the Countable Separation Property, each of which is easy to verify. We collect them in the following list:

(1) If $X \subseteq Y$ and $Y$ has CSP, then $X$ has CSP.

(2) If $S \subseteq E^{0}$ does not have CSP and $\left\{S_{i} \mid i \in \mathbb{N}\right\}$ is a countable set of subsets of $S$, for which each $S_{i}$ has CSP, then $S \backslash \bigcup_{i \in \mathbb{N}} S_{i}$ does not have CSP.

(3) If $S$ is the union of a countable number of subsets of $E^{0}$ each of which has CSP, then $S$ has CSP. 
(4) If $S \subseteq \bigcup_{i \in \mathbb{N}} X_{i}$ and $S$ does not have CSP, then $X_{i}$ does not have CSP for some $i \in \mathbb{N}$.

(5) If $S \subseteq Y \cup Z$ and $Y$ has CSP, then $S$ does not have CSP if and only if $Z$ does not have CSP.

Our approach is as follows. Let $R$ denote $L_{K}(E)$, assume that $R$ is prime, and let $R_{1}$ denote a prime unital ring into which $R$ embeds as a two-sided ideal. We show that, in the absence of the Countable Separation Property on $E^{0}$, the collection $\left\{R_{1} v R_{1} \mid v \in E^{0}\right\}$ of two-sided ideals of $R_{1}$ is "sparse", in the sense that if all of the ideals in this collection are comaximal with a left ideal $I$ of $R_{1}$, then necessarily $I=R_{1}$. This conclusion, combined with Proposition 3.3 , will show that $R_{1}$, and thereby $R$, is not primitive. We utilize the following simple but important observation to draw such a conclusion.

Lemma 5.2. Let $I$ be a left ideal of a unital ring $A$. If there exist $x, y \in A$ such that $1+x \in I, 1+y \in I$, and $x y=0$, then $I=A$.

Proof. Since $1+y \in I$, then $x(1+y)=x+x y=x \in I$, so that $1=(1+x)-x \in I$.

Lemma 5.3. Let $E$ be a graph and let $T$ be a subset of $E^{0}$ which does not have the Countable Separation Property. Suppose that we can associate with each $v \in T$ an element $x_{v} \in L_{K}(E)$ in such a way that, for each $v \in T$, the set

$$
Z(T, v)=\left\{w \in T \mid x_{v} x_{w}=0\right\}
$$

has CSP. Then the set

$$
T^{\sim}=\left\{v \in T \mid\left\{w \in T \mid x_{v} x_{w} \neq 0\right\} \text { does not have CSP }\right\}
$$

does not have CSP.

Proof. Suppose to the contrary that $T^{\sim}$ has CSP. Then $T^{\prime}:=T \backslash T^{\sim}$ does not have CSP, and so in particular is nonempty. Now fix $v \in T^{\prime}$. Then clearly

$$
T^{\prime}=\left\{w \in T^{\prime} \mid x_{v} x_{w} \neq 0\right\} \cup\left\{w \in T^{\prime} \mid x_{v} x_{w}=0\right\} .
$$

As $v \notin T^{\sim}$, the first displayed set has CSP. So, since $T^{\prime}$ does not have CSP, the second displayed set does not have CSP. But this contradicts the hypothesis that $Z(T, v)$ has CSP for all $v \in T$.

The following result will yield the fundamental relationship between the Countable Separation Property in $E^{0}$ on the one hand, and the form of various sets of elements of $L_{K}(E)$ on the other.

Proposition 5.4. Let $E$ be an arbitrary graph, and $K$ any field. Let $S$ be a subset of $E^{0}$ which does not have CSP. Let $d$ be a fixed positive integer, and let $\left(m_{1}, \ldots, m_{d}\right)$ and $\left(n_{1}, \ldots, n_{d}\right)$ be a fixed pair of sequences of nonnegative integers of length $d$. Let $[d]$ denote the set $\{1,2, \ldots, d\}$. Suppose we can associate with each element $v \in S$ an element $x_{v}$ of $L_{K}(E)$ of the form

$$
x_{v}=\sum_{i=1}^{d} k_{i, v} a_{i, v} b_{i, v}^{*}
$$

for which, for all $v \in S$ and $i \in[d]$ :

(i) the expression $\sum_{i=1}^{d} k_{i, v} a_{i, v} b_{i, v}^{*}$ is in standard form (that is, $k_{i, v} \in K$, and $a_{i, v}, b_{i . v}$ are paths in $E$ for which $\left.r\left(a_{i, v}\right)=r\left(b_{i . v}\right)\right)$, 
(ii) the length $\ell\left(a_{i, v}\right)$ of $a_{i, v}$ is $m_{i}$,

(iii) the length $\ell\left(b_{i, v}\right)$ of $b_{i, v}$ is $n_{i}$, and

(iv) $v \geq r\left(a_{i, v}\right) \quad$ (so that $v \geq r\left(b_{i, v}\right)$ as well).

Then there exists $v$ in $S$ for which the set

$$
Z(S, v)=\left\{w \in S \mid x_{v} x_{w}=0\right\}
$$

does not have CSP.

Proof. Suppose not. Then for each $v \in S$, we have that the set $Z(S, v)$ has CSP. Indeed, then, this same property passes to any subset $T \subseteq S$; that is, if $T \subseteq S$, then we have that for each $v \in T$ the set

$$
Z(T, v)=\left\{w \in T \mid x_{v} x_{w}=0\right\}
$$

has CSP. If in addition the subset $T$ of $S$ does not have CSP, then Lemma 5.3 applies to $T$, so that the set

$$
T^{\sim}=\left\{v \in T \mid\left\{w \in T \mid x_{v} x_{w} \neq 0\right\} \text { does not have CSP }\right\}
$$

does not have CSP.

Fix $v \in T^{\sim}$. Now $x_{v} x_{w} \neq 0$ implies that there exists at least one pair $(i, j) \in[d]^{2}$ for which $b_{i, v}^{*} a_{j, w} \neq 0$, so that

$$
\left\{w \in T \mid x_{v} x_{w} \neq 0\right\}=\bigcup_{(i, j) \in[d]^{2}}\left\{w \in T \mid x_{v} x_{w} \neq 0 \text { and } b_{i, v}^{*} a_{j, w} \neq 0\right\} .
$$

Thus

$$
T^{\sim} \subseteq \bigcup_{(i, j) \in[d]^{2}}\left\{v \in T \mid\left\{w \in T \mid x_{v} x_{w} \neq 0 \text { and } b_{i, v}^{*} a_{j, w} \neq 0\right\} \text { does not have CSP }\right\},
$$

a finite union of sets. Then by Remark 5.1 we have that there exists $(i, j) \in[d]^{2}$ for which

$$
\left\{v \in T \mid\left\{w \in T \mid x_{v} x_{w} \neq 0 \text { and } b_{i, v}^{*} a_{j, w} \neq 0\right\} \text { does not have CSP }\right\}
$$

does not have CSP. To summarize: assuming that the set $T^{\sim}$ does not have CSP, we are led to an element of $[d]^{2}$, which we denote by $\left(i_{T}, j_{T}\right)$, for which

$$
\left\{v \in T \mid\left\{w \in T \mid x_{v} x_{w} \neq 0 \text { and } b_{i_{T}, v}^{*} a_{j_{T}, w} \neq 0\right\} \text { does not have CSP }\right\}
$$

does not have CSP.

Now let $\mathcal{S}$ denote the collection of subsets $T$ of the original set $S$ for which $T$ does not have CSP. Then the above discussion yields that there exists a (not necessarily unique) map

$$
\pi: \mathcal{S} \rightarrow[d]^{2}
$$

where $\pi(T)=\left(i_{T}, j_{T}\right)$ such that

$$
\left\{v \in T \mid\left\{w \in T \mid x_{v} x_{w} \neq 0 \text { and } b_{i_{T}, v}^{*} a_{j_{T}, w} \neq 0\right\} \text { does not have CSP }\right\}
$$

does not have CSP.

We now recursively define a countable collection of nested subsets

$$
S=S_{0} \supseteq S_{1} \supseteq S_{2} \supseteq \cdots,
$$

none of which has CSP. We take $S_{0}=S$. Then $S_{0}$ does not have CSP by hypothesis. Having defined $S_{n}$ for $n \geq 0$, we note that there exist $i_{S_{n}}$ and $j_{S_{n}}$ in $\{1, \ldots, d\}$ such that $\pi\left(S_{n}\right)=\left(i_{S_{n}}, j_{S_{n}}\right)$. That is, the set

$\left(S_{n}\right)_{i_{S_{n}}, j_{S_{n}}}^{\sim}=\left\{v \in S_{n} \mid\left\{w \in S_{n} \mid x_{v} x_{w} \neq 0\right.\right.$ and $\left.b_{i_{S_{n}}, v}^{*} a_{j_{S_{n}}, w} \neq 0\right\}$ does not have CSP $\}$ 
does not have CSP. In this case, we pick any $v \in\left(S_{n}\right)_{i_{S_{n}}, j_{S_{n}}}$. (Such $v \in S_{n}$ exists since the set $\left(S_{n}\right)_{i_{S_{n}}, j_{S_{n}}}$ of such $v$ does not have CSP, and thus is nonempty.) If $b_{i_{S_{n}}, v}^{*} a_{j_{S_{n}}, w} \neq 0$, then either $b_{i_{S_{n}}, v}$ is an initial subpath of $a_{j_{S_{n}}, w}$, or $a_{j_{S_{n}}, w}$ is an initial subpath of $b_{i_{S_{n}}, v}$. Now define the set

$$
X=\left\{w \in S_{n} \mid x_{v} x_{w} \neq 0 \text { and } a_{{j_{n}}_{n}, w} \text { is an initial subpath of } b_{i_{S_{n}}, v}\right\} .
$$

(Note that $X$ depends on $n, v, i_{S_{n}}$, and $j_{S_{n}}$.) We claim that $X$ has CSP. To see this, recall that, by hypothesis, $w \geq r\left(a_{{S_{n}}_{n}, w}\right)$. But $r\left(a_{j_{S_{n}}, w}\right) \geq r\left(b_{i_{S_{n}}, v}\right)$ since $a_{{S_{n}}_{n}, w}$ is an initial subpath of $b_{i_{S_{n}}, v}$. So we have that $w \geq r\left(b_{i_{S_{n}}, v}\right)$ for all $w \in X$, which yields that $X$ has CSP with respect to the singleton set $\left\{r\left(b_{i_{S_{n}}, v}\right)\right\}$.

Now $\left\{w \in S_{n} \mid x_{v} x_{w} \neq 0\right.$ and $\left.b_{i_{S_{n}}, v}^{*} a_{j_{S_{n}}, w} \neq 0\right\}$ is the union of the two sets

$$
\left\{w \in S_{n} \mid x_{v} x_{w} \neq 0 \text { and } b_{i_{S_{n}}, v} \text { is an initial subpath of } a_{j_{S_{n}}, w}\right\} \cup X \text {. }
$$

Since $X$ was demonstrated in the previous paragraph to have CSP, we get that, for any $v \in\left(S_{n}\right)_{i_{S_{n}}, j_{S_{n}}}$, the set

$$
S_{n}^{\prime}(v)=\left\{w \in S_{n} \mid x_{v} x_{w} \neq 0 \text { and } b_{i_{S_{n}}, v} \text { is an initial subpath of } a_{{j_{n}}_{n}, w}\right\}
$$

does not have CSP.

We record for later use that in particular this yields that the set $\left(S_{n}\right)_{i_{S_{n}}, j_{S_{n}}}$ defined previously in fact equals the set

$$
\begin{array}{r}
S_{n}^{\prime}=\left\{v \in S_{n} \mid\left\{w \in S_{n} \mid x_{v} x_{w} \neq 0 \text { and } b_{i_{S_{n}}, v} \text { is an initial subpath of } a_{{j_{S_{n}}}_{1}}\right\}\right. \\
\text { does not have CSP }\},
\end{array}
$$

and so $S_{n}^{\prime}$ does not have CSP, and so in particular is nonempty.

Now pick any $v_{n} \in\left(S_{n}\right)_{i_{S_{n}}, j_{S_{n}}}$, and define $S_{n+1}$ by setting

$S_{n+1}=S_{n}^{\prime}\left(v_{n}\right)=\left\{w \in S_{n} \mid x_{v_{n}} x_{w} \neq 0\right.$, and $b_{i_{S_{n}}, v_{n}}$ is an initial subpath of $\left.a_{j_{S_{n}}, w}\right\}$.

By definition, $S_{n+1}$ does not have CSP. We note here the following: for each $n+1$, there exists a path $b_{n+1}=b_{i_{S_{n}}, v_{n}}$ such that $a_{{S_{S}}_{n}, w}$ has $b_{n+1}$ as an initial subpath for all $w \in S_{n+1}$. Moreover, $\ell\left(b_{n+1}\right)=n_{i_{S_{n}}}$ and is in particular independent of the choice of $v_{n}$.

Thus we have defined the sequence

$$
S=S_{0} \supseteq S_{1} \supseteq S_{2} \supseteq \cdots
$$

in such a way that no $S_{n}$ has CSP.

Since the range of $\pi$ is finite, we see that there exist natural numbers $f$ and $g$ with $f<g$ such that $\pi\left(S_{f}\right)=\pi\left(S_{g}\right)=(i, j)$ for some pair $(i, j)$ in $[d]^{2}$. We show that this leads to a contradiction, which will establish the result.

So we have $i_{S_{f}}=i_{S_{g}}=i$ and $j_{S_{f}}=j_{S_{g}}=j$. By the above discussion, there exists a path $b_{f+1}$ of length $n_{i}$ for which $b_{f+1}$ is an initial subpath of $a_{j, w}$ for all $w \in S_{f+1}$.

Since $S_{f+1} \supseteq S_{g+1}$, this property holds for all elements of $S_{g+1}$ as well. But we know that there exists a path $b_{g+1}$ of length $n_{i}$ (n.b.: the same $n_{i}$ as in the previous paragraph) for which $b_{g+1}$ is an initial subpath of $a_{j, w}$ for all $w \in S_{g+1}$. So, since the lengths of each of $b_{g+1}$ and $b_{f+1}$ are equal (to $n_{i}$ ), and each is the initial subpath of a common path (any $a_{j, w}$ for $w \in S_{g+1}$ ), we have that the paths $b_{g+1}$ and $b_{f+1}$ are in fact equal; we denote this common path by $b$. 
Note that the set $S_{g}^{\prime}$ defined above can be written as

$S_{g}^{\prime}=\left\{v \in S_{g} \mid\left\{w \in S_{g} \mid x_{v} x_{w} \neq 0\right.\right.$, and $b_{i, v}$ is an initial subpath of $\left.a_{j, w}\right\}$ does not have CSP\}.

Recall also that $S_{g}^{\prime}$ does not have CSP.

We claim that

$$
S_{g}^{\prime} \subseteq\left\{v \in S_{g} \mid b_{i, v}=b\right\} .
$$

So suppose $v \in S_{g}^{\prime}$; we must show that $b_{i, v}=b$. Since the set

$$
\left\{w \in S_{g} \mid x_{v} x_{w} \neq 0 \text {, and } b_{i, v} \text { is an initial subpath of } a_{j, w}\right\}
$$

does not have CSP, it is in particular nonempty, so let $w \in S_{g}$ have the property that $x_{v} x_{w} \neq 0$, and $b_{i, v}$ is an initial subpath of $a_{j, w}$. Since $w \in S_{g} \subseteq S_{f+1}$, we have by a previous observation that $a_{j, w}$ has $b$ as an initial subpath. But the length of $b_{i, v}$ is $n_{i}$ by definition, which equals the length of $b$, so that $b_{i, v}=b$, thus establishing the claim.

Since $v \geq r\left(b_{i, v}\right)$ for all $v \in S$ by hypothesis, we see that the set $\left\{v \in S_{g} \mid b_{i, v}=b\right\}$ has CSP with respect to the singleton set $\{r(b)\}$. But this leads us to the desired contradiction, since the last displayed inclusion would yield a set without CSP as a subset of a set having CSP, which is impossible by Remark 5.1. This contradiction completes the proof.

The next result indicates that the conditions imposed in Proposition 5.4 are in fact quite natural in the context of two-sided ideals of $L_{K}(E)$ generated by vertices of $E$.

Lemma 5.5. Let $E$ be an arbitrary graph, and $K$ any field. Let $v \in E^{0}$, and let $\langle v\rangle$ denote the ideal $L_{K}(E) v L_{K}(E)$ of $L_{K}(E)$. Then any $0 \neq x \in\langle v\rangle$ can be written in standard form as $x=\sum_{i=1}^{n} k_{i} a_{i} b_{i}^{*}$, where $v \geq r\left(a_{i}\right)=r\left(b_{i}\right)$ for all $1 \leq i \leq n$.

Proof. By definition, $x$ is a $K$-linear combination of nonzero terms of the form $\alpha \beta^{*} v \gamma \delta^{*}$, where $\alpha, \beta, \gamma, \delta$ are paths in $E$. Moreover, $\beta^{*} v \gamma \neq 0$ implies $v=s(\beta)=$ $s(\gamma)$, and either $\beta=\gamma \beta^{\prime}$ or $\gamma=\beta \gamma^{\prime}$ for some paths $\beta^{\prime}, \gamma^{\prime}$ in $E$. With this, the expression $\alpha \beta^{*} v \gamma \delta^{*}$ simplifies to a term of the form $a b^{*}$, and it is readily seen that $v \geq r(a)=r(b)$.

Here is the key result regarding the nonprimitivity of Leavitt path algebras.

Proposition 5.6. Let $E$ be an arbitrary graph, and $K$ any field. If $E^{0}$ does not have the Countable Separation Property, then $L_{K}(E)$ is not primitive.

Proof. If $L_{K}(E)$ is not prime, then the result is immediate. So we may suppose that $L_{K}(E)$ is prime. Let $A$ denote the unital overring $L_{K}(E)_{1}$ of $L_{K}(E)$ as described in Lemma 3.1. Just suppose $A$ is primitive; we seek a contradiction. So by Proposition 3.3 there is a proper left ideal $I$ of $A$ such that $I+J=A$ for every nonzero two-sided ideal $J$ of $A$. Consequently, for each vertex $v \in E^{0}$, there exists some $x_{v} \in A v A$ such that $1+x_{v} \in I$. Since $A v A=L_{K}(E) v L_{K}(E)$, we have that each $x_{v}$ can be written as an element of the form indicated in Lemma 5.5. With this in mind, we define

$$
\Gamma=\bigcup_{d=1}^{\infty}\{d\} \times\left(\mathbb{Z}_{\geq 0}^{d}\right) \times\left(\mathbb{Z}_{\geq 0}^{d}\right),
$$


and for each $\gamma=\left(d,\left(m_{1}, m_{2}, \ldots, m_{d}\right),\left(n_{1}, \ldots, n_{d}\right)\right) \in \Gamma$, we set

$$
\begin{aligned}
S_{\gamma}:=\left\{v \in E^{0} \mid x_{v}=\sum_{i=1}^{d} k_{i} a_{i} b_{i}^{*},\right. \\
\left.\quad k_{i} \in K, v \geq r\left(a_{i}\right)=r\left(b_{i}\right), \ell\left(a_{i}\right)=m_{i}, \text { and } \ell\left(b_{i}\right)=n_{i} \quad \forall i \in[d]\right\} .
\end{aligned}
$$

(We note that the $S_{\gamma}$ need not be disjoint.) By Lemma 5.5 we have that $\bigcup_{\gamma \in \Gamma} S_{\gamma}=$ $E^{0}$. But $\Gamma$ is countable, so since by hypothesis $E^{0}$ does not have CSP, we get that there is some $\gamma \in \Gamma$ such that $S_{\gamma}$ does not have CSP. We now apply Proposition 5.4 to the set $S_{\gamma}$ to conclude that there exists $v \in S_{\gamma}$ such that

$$
Z\left(S_{\gamma}, v\right)=\left\{w \in S_{\gamma} \mid x_{v} x_{w}=0\right\}
$$

does not have CSP. In particular $Z\left(S_{\gamma}, v\right)$ is nonempty, so choose some $w \in Z\left(S_{\gamma}, v\right)$. Then the elements $x_{v}$ and $x_{w}$ of $L_{K}(E)$ corresponding to the vertices $v$ and $w$ respectively have the property that

$$
1+x_{v} \in I, 1+x_{w} \in I, \text { and } x_{v} x_{w}=0 .
$$

Thus by Lemma 5.2, we see that $I=A$, a contradiction.

Thus $A$ is not primitive, and so $L_{K}(E)$ is not primitive by Lemma 3.1

We now have all the pieces in place to establish our main result.

Theorem 5.7. Let $E$ be an arbitrary graph, and $K$ any field. Then $L_{K}(E)$ is primitive if and only if

(i) $E$ is downward directed,

(ii) E satisfies Condition (L), and

(iii) $E^{0}$ has the Countable Separation Property.

Proof. That the three conditions are sufficient is established in Proposition 4.8, while the necessity of downward directedness and Condition (L) was established in the proof of Theorem 3.5 (the row-finiteness hypothesis is not used in those portions of that proof).

The final piece of the proof of Theorem 5.7 (i.e., the necessity of Countable Separation Property) now follows directly from Proposition 5.6.

\section{Prime noprimitive VOn NeUmann REgular RINGS}

As mentioned in the Introduction, for many years Kaplansky's query "Is a regular prime ring necessarily primitive?" was regarded as the major question in the theory of von Neumann regular rings. Theorem 5.7 will allow us to identify various infinite classes of rings for which the answer to Kaplansky's question is No.

Of great use in this context is the fact that the von Neumann regular Leavitt path algebras have been identified.

Theorem 6.1 ([2, Theorem 1]). Let $K$ be any field, and let $E$ be an arbitrary graph. Then $L_{K}(E)$ is von Neumann regular if and only if $E$ is acyclic. In this situation, $L_{K}(E)$ is a directed union of subalgebras, each of which is isomorphic to a finite direct sum of finite-sized matrix rings over $K$.

We note a structural property of the Leavitt path algebras previously defined. 
Lemma 6.2. Let $K$ be any field.

(1) Let $X$ be an infinite set, let $|X|$ denote the cardinality of $X$, and let $E_{\mathcal{F}(X)}$ be the graph described in Definition 4.3 . Then $\operatorname{dim}_{K}\left(L_{K}\left(E_{\mathcal{F}(X)}\right)\right)=|X|$.

(2) Let $\kappa$ be any infinite ordinal, let $|\kappa|$ denote the cardinality of $\kappa$, and let $E_{\kappa}$ be the graph described in Definition 4.5. Then $\operatorname{dim}_{K}\left(L_{K}\left(E_{\kappa}\right)\right)=|\kappa|$.

Proof. (1) Since $|X|$ is infinite, the cardinality of the set $\mathcal{F}(X)$ of finite subsets of $X$ is $|X|$. Thus the graph $E_{\mathcal{F}(X)}$ contains $|X|$ vertices. This set of vertices, being nonzero orthogonal idempotents of $L_{K}\left(E_{\mathcal{F}(X)}\right)$, are necessarily $K$-linearly independent. So $\operatorname{dim}_{K}\left(L_{K}\left(E_{\mathcal{F}(X)}\right)\right) \geq|X|$.

On the other hand, each vertex of $E_{\mathcal{F}(X)}$ emits $|X|$ edges. Since by definition a path in $E$ is a finite sequence of edges, and there are $|X|$ vertices in $E_{\mathcal{F}(X)}$, this yields that there are $|X|$ distinct paths in $E$, so that there are $|X|$ expressions of the form $p q^{*}$ in $L_{K}(E)$ (where $p$ and $q$ are paths in $E$ ). But for any Leavitt path algebra, the set $\left\{p q^{*} \mid p, q\right.$ are paths in $\left.E\right\}$ spans $L_{K}(E)$ as a $K$-vector space. Thus $\operatorname{dim}_{K}\left(L_{K}\left(E_{\mathcal{F}(X)}\right)\right) \leq|X|$, which yields the result.

The proof of (2) is similar.

Proposition 6.3. Let $K$ be any field. Let $X$ be any nonempty set, and let $E_{\mathcal{F}(X)}$ be the graph presented in Definition 4.3. Then $L_{K}\left(E_{\mathcal{F}(X)}\right)$ is a prime ring. Moreover, $L_{K}\left(E_{\mathcal{F}(X)}\right)$ is primitive if and only if $X$ is at most countable.

Proof. Since $E_{\mathcal{F}(X)}$ is clearly acyclic, $E_{\mathcal{F}(X)}$ satisfies Condition (L) vacuously. Since the union of two finite sets is finite, $E_{\mathcal{F}(X)}$ is easily seen to be downward directed. In particular, $L_{K}\left(E_{\mathcal{F}(X)}\right)$ is prime by Theorem 2.4. But by Proposition 4.4, $E^{0}$ has CSP precisely when $X$ is at most countable. The result now follows from Theorem 5.7 .

Here is the first of the previously mentioned classes of prime, nonprimitive, von Neumann regular algebras.

Theorem 6.4. Let $K$ be any field. Let $X$ be an uncountable set, and let $E_{\mathcal{F}(X)}$ be the graph presented in Definition 4.3 . Then $L_{K}\left(E_{\mathcal{F}(X)}\right)$ is a prime, nonprimitive, von Neumann regular ring.

In particular, let $\left\{X_{\alpha} \mid \alpha \in \mathcal{A}\right\}$ denote a collection of uncountable sets, each of different cardinality. Then the collection $\left\{L_{\mathbb{C}}\left(E_{\mathcal{F}\left(X_{\alpha}\right)}\right) \mid \alpha \in \mathcal{A}\right\}$ consists of pairwise nonisomorphic, prime, nonprimitive, von Neumann regular $\mathbb{C}$-algebras.

Proof. By Theorem 6.1, each $L_{\mathbb{C}}\left(E_{\mathcal{F}\left(X_{\alpha}\right)}\right)$ is von Neumann regular. The first statement now follows from Proposition 6.3 That any two such algebras are nonisomorphic follows from Lemma 6.2. (Indeed, we note that in this case these algebras are in fact not even isomorphic as rings, by a similar cardinality argument.)

In a completely analogous manner, we achieve as well the second aforementioned class of prime, nonprimitive, von Neumann regular algebras.

Theorem 6.5. Let $K$ be any field. Let $\kappa$ be an ordinal with uncountable cofinality, and let $E_{\kappa}$ be the graph presented in Definition 4.5. Then $L_{K}\left(E_{\kappa}\right)$ is a prime, nonprimitive, von Neumann regular ring.

In particular, let $\left\{\kappa_{\alpha} \mid \alpha \in \mathcal{A}\right\}$ denote a collection of uncountable ordinals, each of uncountable cofinality, and each having different cardinality. Then the collection $\left\{L_{\mathbb{C}}\left(E_{\kappa_{\alpha}}\right) \mid \alpha \in \mathcal{A}\right\}$ consists of pairwise nonisomorphic, prime, nonprimitive, von Neumann regular $\mathbb{C}$-algebras. 
Remark 6.6. We note that the algebras described in Theorems 6.4 and 6.5 are different up to isomorphism one from the other; that is, $L_{K}\left(E_{\mathcal{F}(X)}\right) \not L_{K}\left(E_{\kappa}\right)$ for any uncountable set $X$ and any uncountable ordinal $\kappa$. The reason is as follows (see e.g. 27] for further details): since each of the graphs $E_{\mathcal{F}(X)}$ and $E_{\kappa}$ has Condition (K), every ideal in each of the algebras $L_{K}\left(E_{\mathcal{F}(X)}\right)$ and $L_{K}\left(E_{\kappa}\right)$ is graded. By [27, Theorem 5.7], for any graph $E$ the lattice of graded ideals of $L_{K}(E)$ is isomorphic to the lattice $\mathcal{L}_{E}$ of pairs $(H, S)$, where $H$ is a hereditary saturated subset of $E^{0}$ and $S$ is a subset of the breaking vertex set $B_{H}$. It is routine to show that for $E=E_{\mathcal{F}(X)}$ there are $\operatorname{card}(X)$ (proper) maximal elements in $\mathcal{L}_{E}$, while for $F=E_{\kappa}$ there is exactly one (proper) maximal element in $\mathcal{L}_{F}$. Thus the ideal lattices of algebras in the two classes are distinct, and therefore any two such algebras are nonisomorphic.

For those readers whose taste runs more towards unital rings, we now show that the previously offered examples may be slightly and easily modified to produce germane classes of such algebras. Let $K$ be a field and let $R$ be any $K$-algebra (not necessarily with multiplicative identity). The $K$-algebra unitization of $R$ is the $K$-algebra

$$
\widehat{R}=K \oplus R,
$$

with coordinate addition and $K$-scalar action, and with ring multiplication given by setting

$$
(k, r) \cdot(l, s)=(k l, k s+l r+r s)
$$

for any $(k, r),(l, s) \in \widehat{R}$. Then $\widehat{R}$ is a $K$-algebra with identity $(1,0)$, and $R$ embeds as a two-sided algebra ideal of $\widehat{R}$ of codimension 1 , via the map $r \mapsto(0, r)$. Let

$$
T=\{y \in \widehat{R} \mid y(0 \oplus I)=\{(0,0)\} \text { for some nonzero two-sided ideal } I \text { of } R\} .
$$

It is shown in the proof of [19, Lemma 2] that if $R$ is prime, then $T$ is a prime $K$-algebra ideal of $\widehat{R}$, and that $T \cap(0 \oplus R)=\{(0,0)\}$. (The primeness of $R$ is needed to show that $T$ is closed under addition.) Indeed, the unital overring $R_{1}$ of $R$ utilized in previous sections of the current article is precisely the quotient ring $\widehat{R} / T$.

We now show that the $K$-algebra unitizations of appropriate Leavitt path algebras provide a class of examples of unital, prime, nonprimitive, von Neumann regular algebras.

Lemma 6.7. Let $K$ be any field, and $R$ any $K$-algebra.

(1) $R$ is prime and nonunital if and only if $\widehat{R}$ is prime.

(2) $R$ is primitive and nonunital if and only if $\widehat{R}$ is primitive.

(3) $R$ is von Neumann regular if and only if $\widehat{R}$ is von Neumann regular.

Proof. Statement (1) follows from [21, Theorem 28] (or from the more focused statement of [22, Corollary 14]). Statement (2) can be deduced from Lemma 3.1 together with statement (1) of this lemma, or from [22, Corollary 17]. For statement (3), it is easy to show that any ideal of a von Neumann regular ring is itself von Neumann regular. Conversely, since $\widehat{R} / R \cong K$ is von Neumann regular, then the regularity of $R$ yields the regularity of $\widehat{R}$ by a standard argument.

We now produce our third class of prime, nonprimitive, von Neumann regular algebras. 
Theorem 6.8. Let $K$ be any field, and $X$ be an uncountable set. Then $L_{K} \widehat{\left(E_{\mathcal{F}(X)}\right)}$ is a unital, prime, nonprimitive, von Neumann regular ring.

In particular, let $\left\{X_{\alpha} \mid \alpha \in \mathcal{A}\right\}$ denote a collection of uncountable sets, each of different cardinality. Then the collection $\left\{L_{\mathbb{C}} \widehat{\left(E_{\mathcal{F}\left(X_{\alpha}\right)}\right)} \mid \alpha \in \mathcal{A}\right\}$ consists of pairwise nonisomorphic, unital, prime, nonprimitive, von Neumann regular $\mathbb{C}$-algebras.

Proof. Since each $E_{\mathcal{F}\left(X_{\alpha}\right)}$ has infinitely many vertices and is downward directed, we have that $\widehat{L_{K}(E)}$ is prime by Theorem 2.4 and Lemma $6.7(1)$. Since $E_{\mathcal{F}\left(X_{\alpha}\right)}$ is acyclic we get that $L_{K}\left(E_{\mathcal{F}\left(X_{\alpha}\right)}\right)$ is von Neumman regular by [2, Theorem 1], and thus $L_{K} \widehat{\left(E_{\mathcal{F}\left(X_{\alpha}\right)}\right)}$ is von Neumann regular by Lemma 6.7(3). Finally, Lemma 6.7(2) along with Proposition 5.6 yield that $L_{K} \widehat{\left(E_{\mathcal{F}\left(X_{\alpha}\right)}\right)}$ is not primitive.

That the algebras are pairwise nonisomorphic follows from Lemma 6.2 and the fact that $L_{K} \widehat{\left(E_{\mathcal{F}\left(X_{\alpha}\right)}\right)}$ is a one-dimensional extension of $L_{K}\left(E_{\mathcal{F}\left(X_{\alpha}\right)}\right)$.

Arguing in exact analogy to the proof of Theorem 6.8, we achieve our final class of examples.

Theorem 6.9. Let $K$ be any field, and $\kappa$ any ordinal with uncountable cofinality. Then $\widehat{L_{K}\left(E_{\kappa}\right)}$ is a unital, prime, nonprimitive, von Neumann regular ring.

In particular, let $\left\{\kappa_{\alpha} \mid \alpha \in \mathcal{A}\right\}$ denote a collection of uncountable ordinals, each of uncountable cofinality, and each of different cardinality. Then the collection $\left\{\widehat{L_{\mathbb{C}}\left(E_{\kappa_{\alpha}}\right)} \mid \alpha \in \mathcal{A}\right\}$ consists of pairwise nonisomorphic, unital, prime, nonprimitive, von Neumann regular $\mathbb{C}$-algebras.

Remark 6.10. As was done for the two classes of nonunital algebras produced at the beginning of this section, we observe here that the unital algebras described in Theorems 6.8 and 6.9 are different up to isomorphism one from the other; that is, $L_{K} \widehat{\left(E_{\mathcal{F}\left(X_{\alpha}\right)}\right)} \neq \widehat{L_{K}\left(E_{\kappa}\right)}$ for any uncountable set $X$ and any uncountable ordinal $\kappa$. This follows from Remark 6.6 together with the fact that the maximal ideal structure of $\widehat{R}$ is completely determined by the maximal ideal structure of $R$ (see e.g. [22, Corollary 18]).

Immediately after posing the aforementioned "Is a regular prime ring necessarily primitive?" question in [16], Kaplansky continued: "It seems unlikely that the answer is affirmative, but a counter-example may have to be weird." While weirdness is certainly in the eye of the beholder, we believe that the examples of prime nonprimitive regular algebras presented in this section arise quite naturally. Indeed, by Theorem 6.1, every one of these examples is a direct union of subrings, each of which is isomorphic to a finite direct sum of finite-sized matrix rings over the field $K$.

Remark 6.11. In addition to the graphs of the form $E_{\mathcal{F}\left(X_{\alpha}\right)}$ and $E_{\kappa}$ described above, there are many additional classes of graphs germane in the current context. For instance, let $X$ be any infinite set, and let $\mathcal{T}_{X}$ denote the collection of those subsets $A$ of $X$ for which $\operatorname{card}(A)<\operatorname{card}(X)$. We define a graph $E=E_{\mathcal{T}_{X}}$ as follows:

$$
E_{\mathcal{T}_{X}}^{0}=\mathcal{T}_{X}, E_{\mathcal{T}_{X}}^{1}=\left\{e_{A, A^{\prime}} \mid A, A^{\prime} \in \mathcal{T}_{X} \text {, and } A \varsubsetneqq A^{\prime}\right\},
$$

$s\left(e_{A, A^{\prime}}\right)=A$, and $r\left(e_{A, A^{\prime}}\right)=A^{\prime}$ for each $e_{A, A^{\prime}} \in E_{\mathcal{T}_{X}}^{1}$. Then $E$ is acyclic and is downward directed, and $E$ has CSP if and only if $X$ is at most countable. 
Remark 6.12. As mentioned in the Introduction, for a graph $E$ one may define the graph $\mathrm{C}^{*}$-algebra $C^{*}(E)$. In a suitable topology, the Leavitt path algebra $L_{\mathbb{C}}(E)$ may be viewed as a dense $*$-subalgebra of $C^{*}(E)$. There are examples of prime nonprimitive graph $\mathrm{C}^{*}$-algebras which appear in the literature; see e.g. [17, Proposition 13.4]. However, these examples are not germane in the context of Kaplansky's question, as one can show that $C^{*}(E)$ is not von Neumann regular whenever $E$ is an acyclic graph having infinitely many vertices.

We conclude with the following observation. For any graph $E$ and field $K$ the Cohn path algebra $C_{K}(E)$ is the $K$-algebra having the same generators and relations as those given in Definition 2.1 for the Leavitt path algebra $L_{K}(E)$, except for the (CK2) relation. (The Cohn path algebras are the natural generalizations to graphs of the algebras $U_{1, n}$ defined in [9, Section 5].) We note that for graphs $E$ of the form $E_{\mathcal{F}(X)}$ or $E_{\kappa}$, the Leavitt path algebra $L_{K}(E)$ and Cohn path algebra $C_{K}(E)$ coincide, as each vertex $v$ in $E$ has $\left|s^{-1}(v)\right|=\infty$. Thus the examples of prime nonprimitive von Neumann regular rings given in Theorems 6.4 and 6.5 may be interpreted as arising from the Cohn path algebra construction as well.

\section{ACKNOWLEDGEMENT}

The authors are profoundly grateful to the referee for an extremely careful reading of the original manuscript, as well as for the numerous suggestions which led to a significantly improved revised version.

\section{REFERENCES}

[1] G. Abrams and G. Aranda Pino, The Leavitt path algebras of arbitrary graphs, Houston J. Math. 34 (2008), no. 2, 423-442. MR2417402 (2009h:16043)

[2] Gene Abrams and Kulumani M. Rangaswamy, Regularity conditions for arbitrary Leavitt path algebras, Algebr. Represent. Theory 13 (2010), no. 3, 319-334, DOI 10.1007/s10468008-9125-2. MR2630124 (2011b:16108)

[3] Pere Ara and Miquel Brustenga, The regular algebra of a quiver, J. Algebra 309 (2007), no. 1, 207-235, DOI 10.1016/j.jalgebra.2006.10.013. MR2301238 (2008a:16019)

[4] G. Aranda Pino, D. Martín Barquero, C. Martín González, and M. Siles Molina, The socle of a Leavitt path algebra, J. Pure Appl. Algebra 212 (2008), no. 3, 500-509, DOI 10.1016/j.jpaa.2007.06.001. MR2365329 (2008k:16022)

[5] Gonzalo Aranda Pino, Dolores Martín Barquero, Cándido Martín González, and Mercedes Siles Molina, Socle theory for Leavitt path algebras of arbitrary graphs, Rev. Mat. Iberoam. 26 (2010), no. 2, 611-638, DOI 10.4171/RMI/611. MR.2677009 (2011f:16068)

[6] G. Aranda Pino, E. Pardo, and M. Siles Molina, Exchange Leavitt path algebras and stable rank, J. Algebra 305 (2006), no. 2, 912-936, DOI 10.1016/j.jalgebra.2005.12.009. MR2266860 (2007i:16009)

[7] G. Aranda Pino, E. Pardo, and M. Siles Molina, Prime spectrum and primitive Leavitt path algebras, Indiana Univ. Math. J. 58 (2009), no. 2, 869-890, DOI 10.1512/iumj.2009.58.3516. MR2514392(2010c:16026)

[8] Gonzalo Aranda Pino, Kulumani Rangaswamy, and Lia Vaš, *-regular Leavitt path algebras of arbitrary graphs, Acta Math. Sin. (Engl. Ser.) 28 (2012), no. 5, 957-968, DOI 10.1007/s10114011-0106-8. MR.2909785

[9] P. M. Cohn, Some remarks on the invariant basis property, Topology 5 (1966), 215-228. MR0197511 (33 \#5676)

[10] Joachim Cuntz, Simple $C^{*}$-algebras generated by isometries, Comm. Math. Phys. 57 (1977), no. 2, 173-185. MR0467330 (57 \#7189)

[11] O. I. Domanov, A prime but not primitive regular ring, Uspehi Mat. Nauk 32 (1977), no. 6(198), 219-220 (Russian). MR0573043 (58 \#28058) 
[12] Carl Faith, Rings and things and a fine array of twentieth century associative algebra, Mathematical Surveys and Monographs, vol. 65, American Mathematical Society, Providence, RI, 1999. MR1657671 (99j:01015)

[13] Joe W. Fisher and Robert L. Snider, Prime von Neumann regular rings and primitive group algebras, Proc. Amer. Math. Soc. 44 (1974), 244-250. MR0342551 (49 \#7297)

[14] Edward Formanek, Group rings of free products are primitive, J. Algebra 26 (1973), 508-511. MR0321964 (48 \#329)

[15] K. R. Goodearl, Leavitt path algebras and direct limits, Rings, modules and representations, Contemp. Math., vol. 480, Amer. Math. Soc., Providence, RI, 2009, pp. 165-187, DOI 10.1090/conm/480/09374. MR2508151 (2010h:16073)

[16] Irving Kaplansky, Algebraic and analytic aspects of operator algebras, American Mathematical Society, Providence, R.I., 1970. Conference Board of the Mathematical Sciences Regional Conference Series in Mathematics, No. 1. MR0312283(47 \#845)

[17] Takeshi Katsura, A class of $C^{*}$-algebras generalizing both graph algebras and homeomorphism $C^{*}$-algebras. III. Ideal structures, Ergodic Theory Dynam. Systems 26 (2006), no. 6, 18051854, DOI 10.1017/S0143385706000320. MR2279267 (2007j:46090)

[18] T. Y. Lam, A first course in noncommutative rings, 2nd ed., Graduate Texts in Mathematics, vol. 131, Springer-Verlag, New York, 2001. MR.1838439 (2002c:16001)

[19] Charles Lanski, Richard Resco, and Lance Small, On the primitivity of prime rings, J. Algebra 59 (1979), no. 2, 395-398, DOI 10.1016/0021-8693(79)90135-2. MR543258(80i:16009)

[20] W. G. Leavitt, The module type of a ring, Trans. Amer. Math. Soc. 103 (1962), 113-130. MR.0132764(24 \#A2600)

[21] Zachary Mesyan, The ideals of an ideal extension, J. Algebra Appl. 9 (2010), no. 3, 407-431, DOI 10.1142/S0219498810003999. MR2659728(2011g:16049)

[22] Z. MESYAN, Ideal extensions of simple rings, preprint.

[23] C. Năstăsescu and F. van Oystaeyen, Graded ring theory, North-Holland Mathematical Library, vol. 28, North-Holland Publishing Co., Amsterdam, 1982. MR676974 (84i:16002)

[24] Donald S. Passman, Group rings of polycyclic groups, Group theory, Academic Press, London, 1984, pp. 207-256. MR780571 (86e:16019)

[25] Iain Raeburn, Graph algebras, CBMS Regional Conference Series in Mathematics, vol. 103, Published for the Conference Board of the Mathematical Sciences, Washington, DC, 2005. MR2135030(2005k:46141)

[26] Louis H. Rowen, Ring theory. Vol. II, Pure and Applied Mathematics, vol. 128, Academic Press Inc., Boston, MA, 1988. MR945718 (89h:16002)

[27] Mark Tomforde, Uniqueness theorems and ideal structure for Leavitt path algebras, J. Algebra 318 (2007), no. 1, 270-299, DOI 10.1016/j.jalgebra.2007.01.031. MR2363133 (2008h:16030)

Department of Mathematics, University of Colorado, Colorado Springs, Colorado 80918

E-mail address: abrams@math.uccs.edu

Department of Mathematics, Simon Fraser University, Burnaby, British Columbia, CANADA V5A1S6

E-mail address: jpb@sfu.ca

Current address: Department of Pure Mathematics, University of Waterloo, 200 University

Avenue W, Waterloo, Ontario, Canada N2L 3G1

E-mail address: jpbell@uwaterloo.ca 80918

Department of Mathematics, University of Colorado, Colorado Springs, Colorado

E-mail address: krangasw@uccs.edu 